\title{
INELASTIC ELECTRON-ION SCATTERING IN A DENSE PLASMA
}

\author{
G. J. hatton ${ }^{\dagger}$, N. F. Lane ${ }^{\dagger}$, and J. C. Weisheit ${ }^{ \pm}$ \\ tDepartment of Physics and Rice Quantum Institute \\ Rice University, Houston, TX 77001 \\ *Plasma Physies Laboratory, Princeton University \\ Princeton, NJ 08544
}

\begin{abstract}
The Born approximation was used to investigate the influence of a jensis plasma on the inelastic scatterigg of electrons by one-electron ions. Scale: collisinn strengths $z^{2}$, for $i s+2 s, 1 s+2 p$ and $2 s+2 p$ transitions in an ion of arhitrary nuclear charge $z$ were computed for a Debye-liuckel morel of the. screened Coulomb interaction. Over a wide range of screening lengths, the effect of the plasma environment is to appreciably reduce cros sections just. above threshold.
\end{abstract}

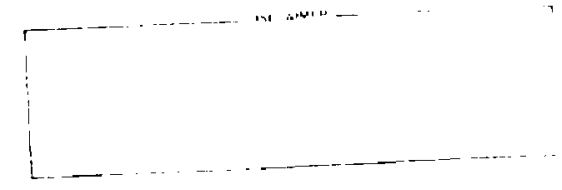




\section{INTRODUCTION}

The interpretation of line emission from dense, high-temperature plasmas, such as those occurring in inertial confinement fusion experiments, rerulres detailed knowledge of spectroscopic and collision properties of ions under extreme conditions (Bekefi et al., 1976; Boiko et al., 1979; ana weishejt, 19811. Some progress has been made in determining the influence of the 51 tim. on atomic structure (Rogers et al., 1970: Rozsnyai, 1972). However, vur: L:ttle is known about plasma effects on important collisional propessess. zross sections for electron impact excitation and ionization of isniate? positive ions are finite at thresuold because of the long-zange Coulomb intera-tion between the collision partners (Massey and Burhop, 1969), f.st. 1 l ? tense piasma partial shielding by nearby charged particlis retuces th:s ejectrostatir interaction at large separations. Therefore, we expect tho effects of plasma screening on collision cross sections to be most sign: finant ir. the near-threshold region. Moreover, it is this energy requin to whils thermal excitation rates ofter are mogt sensitive since, in many importan: instances, thes oxcitation threshold exceeds the plasma electrons' moar. kiriteil energy.

Th1s paper reports the first model calcuiations of linelistir irises

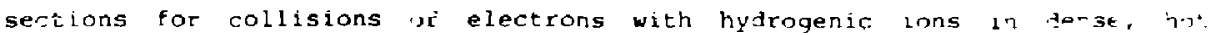
piasmas. In sec. 2 our model is described, and in Secs, 3 arit 4 our resul*s are presenter and discussed. Unless otherwise noted, giuantities are expressed in atomic units.

2. THEORETIT:AL MODEL.

The cross section for the transition ip $+n^{\prime p} p^{\prime}$ in a one-electron lon, Induced by an incident electron having initial kinetic energy $\mathrm{E}=\mathrm{k}^{2}, 2,: 5$ given by (cf. Seaton, 1961) 


$$
\left.Q\left(n q_{a}+n^{\prime} q_{a}^{\prime}\right)=\left(\frac{\pi}{\sqrt{2 q_{a}+1 k^{2}}}\right) \text { of } \ell_{a}, n^{\prime} l_{a}^{\prime}\right) \text {, }
$$

where the collision strength $O$ is defined in terms of he sartial collisions strengths and transition (T) matrix elements,

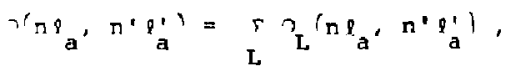

and where

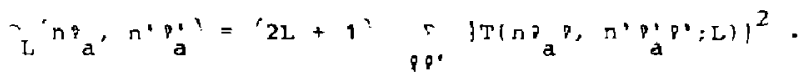

The smali spin-dependent interactions betweer the incident electron and the ion are ignored. so the total orbital anoular momentura $\vec{L}$ is conserved in the roblision. In Eqs. (2) and (3), L takes on all values consistent witl: $t^{3}+t^{\prime}$

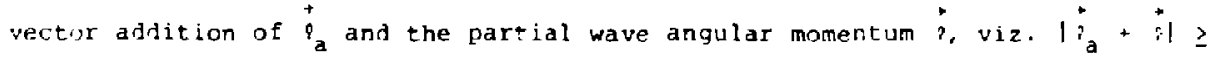

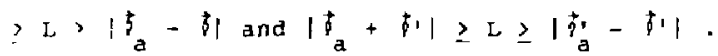

The exchange coutribution to $Q$ is neglerted and the collision problem is formulated in the first Born approximarion. These simplifications permit the series of partial-wave contributions in Eqs. (2) and (3) to be sumed firmally to yield the familiar integral expression for the collision strength,

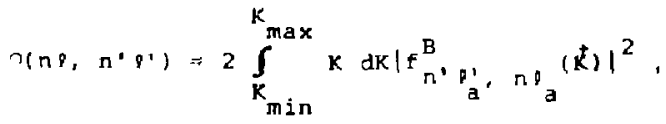


where the momentum change $\vec{k}$ of the scattered electron varies in magnitude from $k_{\min }=\left|\vec{k}-\overrightarrow{k^{\prime}}\right|$ to $x_{\max }=\left|\vec{k}+\overrightarrow{k^{\prime}}\right|$, with $\Delta E\left(n, n^{\prime}\right)=1 / 2\left(k^{2}-k^{\prime 2}\right)$ being th. threshold energy for excitation. The first Born scattering amplitude $f^{B}$ is related to the matrix element of the electron-ion interaction $v$ acording co thit formula

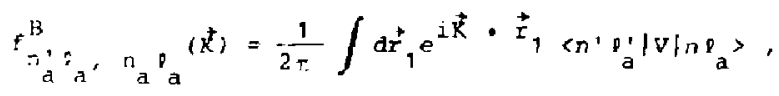

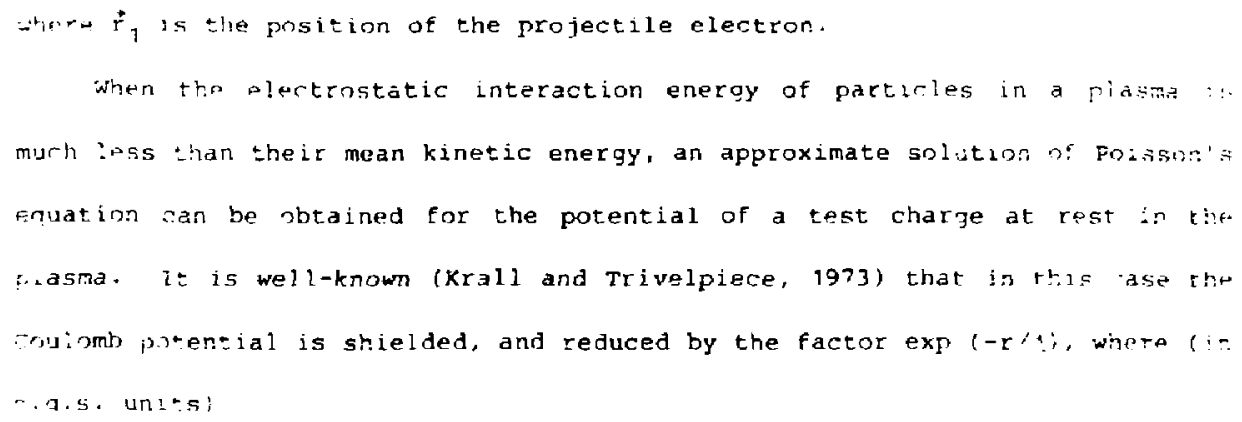

$$
\left.=\frac{4-\frac{4 E^{2}}{k_{B}}}{i} ; N_{j} z_{j}^{2}\right)^{-1 / 2}
$$

is the Deyre-liükel screening length for a plasma of temperature $T$ trat has a density $N_{j}$ of particles with charge $z_{j}$. Here, the test charge is a hydrogenic ion pessiessing some thermal energy. Because of its motion, only the electrans ars generally effoctlve in providing efficint shielding (krall and Trivelpiece, 1973), so that 


$$
\Lambda \rightarrow A_{e}=\left(k_{B} T / 4 \pi e^{2} N_{e}\right)^{1 / 2}
$$

is the screening length apprcpriate to the scattering process.

The Deybe-Hückel appioximation of the interaction is 1nvalin in the limit of high densities and low temperatures. However, even when it is necessary to resort to a full solution of Poisson's equation, it is found (cf. Stewart and Fyatt, 1966) that far from the ion of interest the Deybe-Hückel form is regained. Thus, we expect the exponentially screened electron-ion coulsmb interaction,

$$
v\left(\vec{r}_{1}, \vec{r}_{2}\right)=\left(-\frac{z}{r_{1}}+\frac{1}{\left|\vec{r}_{1}-\vec{r}_{2}\right|} \mid \exp \left(-r_{1} / A\right),\right.
$$

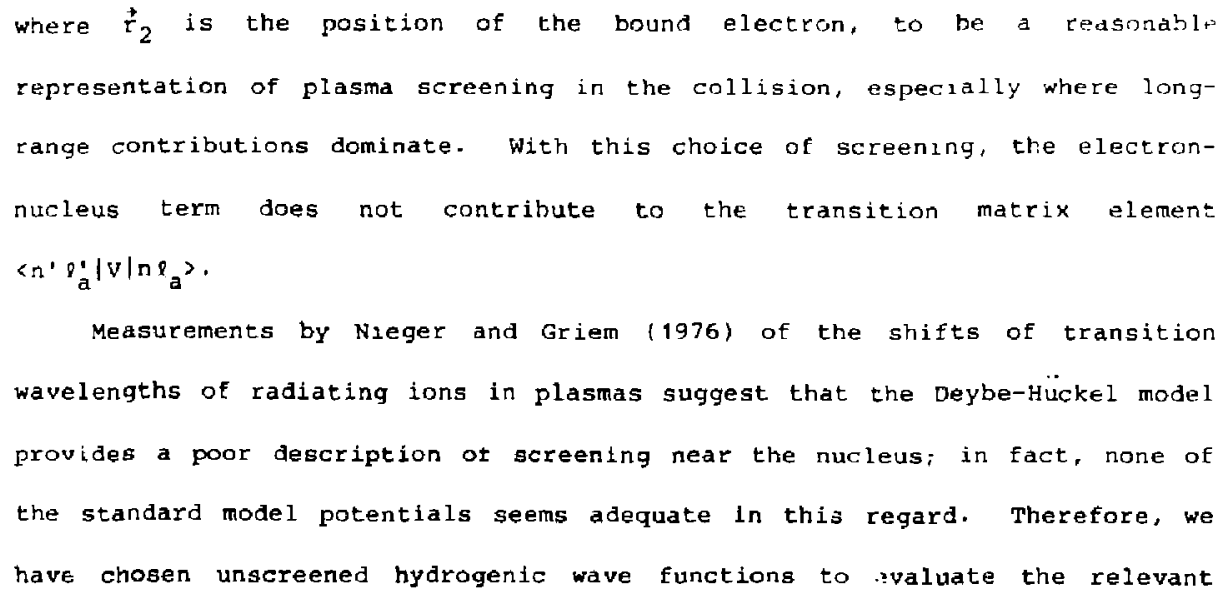


atomic matrix elements for most of the calculations. Besides simplicity, this choice has the added virtue of isolating the collisional effects of a screened coulomb interaction.

The Born approximation to the scattering problem is accurate under conditions ct weak coupling between target states and weak distortion of the scatterea electron wave function. These conditions generally are met for the higher-order partial waves at a given impact energy, or for the totai cross section in the high-energy limit. In consequence, our numerical results far the collisiosn strengths $a$ probably are only qualitatively correct in the region near threshold. We have calculated partial-wave contributions $m_{L}$ as well as total collision strengths in order to get some feeling for the overall validity of the scattering calculations. These computations will be discusser in the next. section.

3. NUMERITAL RESULTS

By caking alvantage of the scaling properties of this collision problem with respert to nuclear charge, namely,

$$
k=k(2)=2 k(1), \Lambda=\Lambda(2)=z^{-1} \Lambda(1), O=D(z)=z^{-2} \cap(1)
$$

we need only to cutry out the calculations for a one-electron "ion" of nuclear charge $z=1$. This scaling is exact for the Bors (not Coulomb Born) approximation, but only approximate in general (Burgess et al., 1970), We report only the collision atrength $O$, since it is a convenient quantity for 1 Jrposes of comparison. In the absence of strong coupling between target 

For Einite screening lengths, the "correct" collision strengths and cross sections will vanish at threshold due to the digtortion of the final-state continuum wave function by the long-range average electron-ion interaction. of course, none of these calculations takes into account resonances iMassey and Burhop, 1969: Burke et al., 1964) which in the weak-gcreening (large : limit should be pregent in abundance. Although a more realisic representation of the plasma-ion interaction might be expected to wash out narrow resonances, they could still influence thermal reaction rate coefficients. Pinally, collision strengths calculated for screened targetstate energies (Rogers et al., 1970) are also given in Figs, 3 and 4. The effects are seen to be relatively small.

The $25+2 p$ collision strength exhibits a strong sensitivity to plasma screening of the electron-ion interaction, as illustrated in Fig. 5. Plasma screening modifies atomic eigenvalues, but even at very high densities the predicted level shifts are small (skupsky, 1980). Lifting the 2s, $2 p$ orbital angular momentum degeneracy of the hydrogenic states by using the correct eigenvalues (Rogers et al., 1970) for the Deybe-Hückel screened ion, we obtain the results plotted as closed circles in Fig. 5. Except just above threshold (not apparent in the figure), there is no noticeable change in $z^{2} \circ(2 s, 2 p)$, in marked contrast to the pure coulomb case (Burgess et al. , 1970).

We also investigated an alternative Debye-Hückel screening model in. which the electron-electron interaction is modified by the non-spherical term $\exp \left(-\left|\vec{r}_{1}-\vec{r}_{2}\right| / \Lambda\right)$; this represents a pic':ure wherein the bound electron carries its own cloud of Bcreening charge. Because the orbital speed of the hound electron is much greater than the speeds of most plasma electrons, in this alternative picture the "relevant" screening length should probably be much greater than $h_{e}$. However, this aslde, the calculations sinow that by 
ignoring tije concentration of charge around the ion as a whole, the net effect of screening is already reduced. For example, the calculated collision strengths for spherical screening are numerically similar to the nonspherical-screening results if the screening length used in the former is about five times that used in the latter.

\section{DISCUSSION}

As mentioned in the introduction, collisional transition rates are needer to interpret line emission from dense plasmas. The general expression for the thermal rate coefficient (i.e., the velocity averaged cross section) for the eroitation $n p^{\prime}+n^{\prime} \rho_{a}$ in a hydrogenic ion of nuclear charge $z$ is

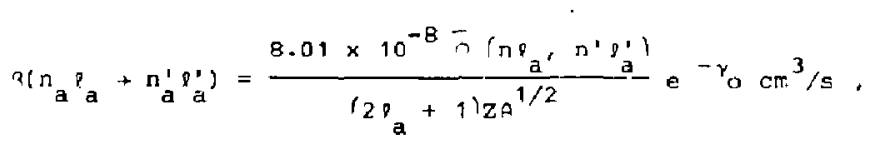

where $\mathrm{z}^{2} \mathrm{~A}(\mathrm{eV})=\mathrm{k}_{\mathrm{B}} \mathrm{T}$ is the plasma temperature in units of electron volts, $\gamma_{0}=$ $A E\left(n l_{a}, n^{\prime} l_{a}^{\prime}\right) / z^{2} \theta$, and

$$
z^{2} \bar{\phi}\left(n \ell_{a}, n^{\prime} \ell_{a}^{\prime}\right)=\int_{0}^{\infty} d y z^{2} O\left(n \ell_{a}, n^{\prime} \ell_{a}^{\prime}\right) e^{-y}
$$

with $x=E / Z^{2} A-x_{0}$ being the ir negration variable.

As an illustration of the influence of plasma screening, consifer the $1 \mathrm{~s}$ $\rightarrow 2 s$ transition, whose unscreened hydrogenic collision strength (Burgess et al., 1973; on et al., 1978), $z^{2} g^{(\mathrm{H})}(1 \mathrm{~s}, 25)=0.45$, for large $z$, is 
essentially independent of energy. The screened hydrogenic collision strengths (for arbitrary z) can be approximated by the formula

$$
z^{2} n(1 s, 2 s)=z^{2} n\left[1-e^{-r(E / \Delta E-1)}\right\}
$$

where $Z^{2} \circ$ is the asymptotic $\{E / A E \gg, 1)$ value and $r$ is an anjustablw parameter. Substitution of this expression into Eq. (11) yields

$$
z^{2} \gamma(15,25)=z^{2} n_{0}\left[\gamma /\left(\gamma+y_{0}\right)\right]
$$

Typically, a given stage of ionization is preduminant in a plasme when its ionization potential is a few times the temperature $z^{2} \mathrm{~A}$. Choosing $y_{0}=3$, we find that plasma screening decreases the excitation rate coefficient $9(1,25)$ to $0.33,0.27$, and 0.23 , times its unscreened value when $z \Lambda=100 a_{0}$, $20 a_{0}$, and $10 a_{o^{\prime}}$ respectively. It is clear from Eigs. 3 and 4 trat the $15+2 p$ transition rate coefficient will be even mare severely reduced at a given zavalue. However, because $x_{0}=0$ for the $2 s \rightarrow 2 p$ transition, plasma screening has a somewhat smaller effect on its collisional rate coefficient.

A specific numerical example is provided by a University of Rochester experiment (Yaakobi et al., 1979) in which a neon-filled glass microballoon was jmploded by interse lager irradiation. Strong resonance lines of hydrogen-like and helium-like neon ions were osserved, and the plasma conditions were estimated to be $\mathrm{N}_{e}=7 \times 10^{22} \mathrm{~cm}^{-3}$ and $\mathrm{z}^{2} \mathrm{~s}=300 \mathrm{ev}$. These 
vaiues yield $x_{0}=3.3$ and $z_{\Lambda_{e}}=92 a_{0}$. Even though nur Born calculations are only qualitatively correct, they suggest that significant reductions of collisional rate coefficlents are likely to result from screening effects in the dense plrsmas now being generated in many laboratories.

In the future, we intena to perform "few-state" close coupling calculations for some screened-Coulomb scattering provesses. Comparison with the Born results should enable us to estimate the izportance of distortion and strong-coupling effects. Some measure of the influence of exchange and correlation effects can be obtained by appropi ately scaling the led-rader pätial-wave collision strengths from accurate bare-ion calculations fsee for example review by Henry, 1980) in a manner consistent with our (plasma) cIosa coupling studies. Other issues to be examined included possible modification of the long-range polarization interaction since plasma screening not oniy reduces the electric field of the incident electron, but also perturbs the ion, thereby changing its polarizabisity $\alpha$. This could affect the threshold behavior of collision cross sections (Gailitis and Damburg, 1963). Another problem concerns the handing of plasma screening for transitions to ai Eroi: innic states with non-zero $\mathrm{p}^{-v a l u e s, ~ t h e ~ s c r e e n i n g ~ f a c t o r ~ e x p l-r / A\} ~ b e i n g ~}$ aporopriate only for spherical charge distributions. If an ton's net electrostatic potential outside the reqion of its v, bound electrons is represented by a multipole expansion, say

$$
\operatorname{tin}_{\text {ion }}\left(t_{1}\right)=\sum_{\lambda=0,2, \ldots}^{2 \ell_{a}}\left[\frac{P_{\lambda}\left(\cos \theta_{1}\right)}{r_{1} \lambda+1} \mu_{\lambda}\left(I_{2}\right)\right],
$$

where $M_{0}=\left\langle z-y_{b}\right\rangle_{\text {, it }}$ can be shown that Deybe-tückel screening reduces each multipole term $M_{\lambda}$ by the factor 


$$
e^{-r_{1} / \Lambda}\left[\sum_{\mu=0}^{\lambda}\left(_{\mu}\right) \frac{(\lambda+\mu) !}{(2 \lambda) !}\left(\frac{2 r_{1}}{\Lambda}\right)^{\lambda-\mu}\right] .
$$

(This formula is valld as long as $\Lambda \gg \Leftrightarrow f$ fective gize of the lon.) While it is stre: rhtforward to include this change in the diagnoal coupli..g matrix elements, it is not clear how an appropriate modification of the off-diagonal coupling matrix elements can be made.

\section{ACKNOWLE.JGMENT}

This work was suppored in part (G.J.H. and N.F.L.) by the U. $S$. Department of Energy, Offise of Basic Energy Sciences and the Robert $A$. Welch Foundation, and in part (J.C.W.) by the U.S. Department of Energy, Contracts DE-ACO2-75-CHO3073 and W-7405-ENG-48. 
REFERENCES

Eekefi G., Deutsch, C., and Yaakoibi, 1976 in "Principles of Laser Plasmas," ed., Bekefi, G. (J. Wiley, New York) 549-641.

Boiko, v. A., Pikuz, S. A., and Faenov, A. Ya., t979, J. Phys. B 12, 18891910.

Burgess, A., Hummer, D. G., and Tully, J. A., 1970, Phil. Trans. Roy, Snc. (London; 22E, 225-279.

Burke, P. G., McVicar, D. D., and Jmith, K., 1964, Phys, Letters 12, $215-216$. Gailitis, M., and Damburg, R., 1963, proc. Phys. 5oc. (London) E2, 19:-200. Henry, R, J, W., 1981, Physics Repocts (to be publishei).

Krall, N. A., and Trivelpiece, A. W., 1973, "Prinaiples of plasma Physics" (MuGraw 4ill, New York) Chapter 11.

Massey, H. S. W., and Burhop, E. H. S., 1969, "Electronic and Isnir Impact Phenomena," Vol. I (The University Press: Oxford) Chapter 7. Neiger, M., and Griem, H. R., 1976, Phys. Rev. A14, 291-299. Oh, S. D., Macek, J., ind Kelsey, E., 1978, Phys. Rev. A17. B73-879. Rogers, F. J., Gratosks, H. J., and harwood, D. J., 1970, Phys. Rev. A1, 15771586.

Rousnyai, B. F., 1972, Phys, Rev. A5, 1137-1149.

Sezton, M. J., 1961, Proc. Phys. Sos, (London) 77, 174-183.

Skupsky, S., 1980, Fhys - Rev. A21, 1316-1326.

Stewart, J. C., and Pyatt, K. D., 1966, Astrophys. J. 144, 1203-1211.

Weisheit J. C., and Shore, B. W., 1974 Astrophys. J. 194, 519-523.

Weisheit, J. C., 1901 in "Applied Atomic Collision Physics," ed., Masssy, H. S. W. (Academic Press, New York) in press.

Yaakobi, B., Steel, D., Thotos, E., Hauer, A., Perry, B., Skupsky, S., Geiger, 
J., Lee, C. M., Letzring, s., Rizzo, J., Mukaiyama, T., Lazarus, E., Halperr., G., Deckman, H., Delettrez, J., Soures, J., and Mccrory, R., 1979, Phys. Re'. A19, $1247-1262$. 
FIGURE CAPTIONS

Fig. 1 Partial collision strengths $z^{2} \eta_{1}$ as a function of $\mathrm{L}$ tor the $1 \mathrm{~s} \cdot 2 \mathrm{~s}$ transition at energy $\mathrm{E} / \mathrm{Z}^{2}=2.25$ a.u. and screening lengths $: Z=$ in, 20. 50 and $100 \mathrm{a}_{0}$.

Fig- 2 Partial collision strengths $\left.z^{2}\right\urcorner_{L}$ as a function of $L$ for the $15+2 \Gamma$ transition at energy $E / Z^{2}=2.25$ a.u. and screening lengtn $: z=10$, 29 and 50 a.

Fig. 3 Total collisions strengths $z^{2}$, as a function of energy E/z $z^{2}$ in a.u.। for the is $+2 \mathrm{~s}$ transition for screening lengths $A 2=10,20,50,100$ and $v$ a $(\longrightarrow$ Results corresponding to screned target-state energies $\overline{\mathrm{r}} 0 \mathrm{r} \mathrm{Z}=10 \mathrm{a}_{0}$ and $20 \mathrm{a}_{0}(-----)$ are above the respectiva unscreened-energies curves. Coulomb Born I ( $B(t)$ results for $z=2$ are also shown.

Fig. 4 Total collision strengths $z^{2} O$ as a function of energy $E / z^{*}($ in a.u. for the $1 s+2 p$ transition For screening lengths $1 z=10,20,50$ and $\infty a_{0}(-)$ Results corresponding to screened target-state energies for $\Lambda z=10$ a (closed circles) are just above the unscreened-energies curve. Coulomb Born I (CBI) results for $\mathrm{Z}=2$ are also shown.

Fig. 5 Total collision strengths $z^{2} n$ as a function of energy $E / z^{2}(i n a \cdot u \cdot)$ for the $2 \mathrm{~s}+2 \mathrm{p}$ transition for screening lengths $\Lambda z=10$ ai. 20 a (-). Results for screened target-state energies are also shown (closes circles). 


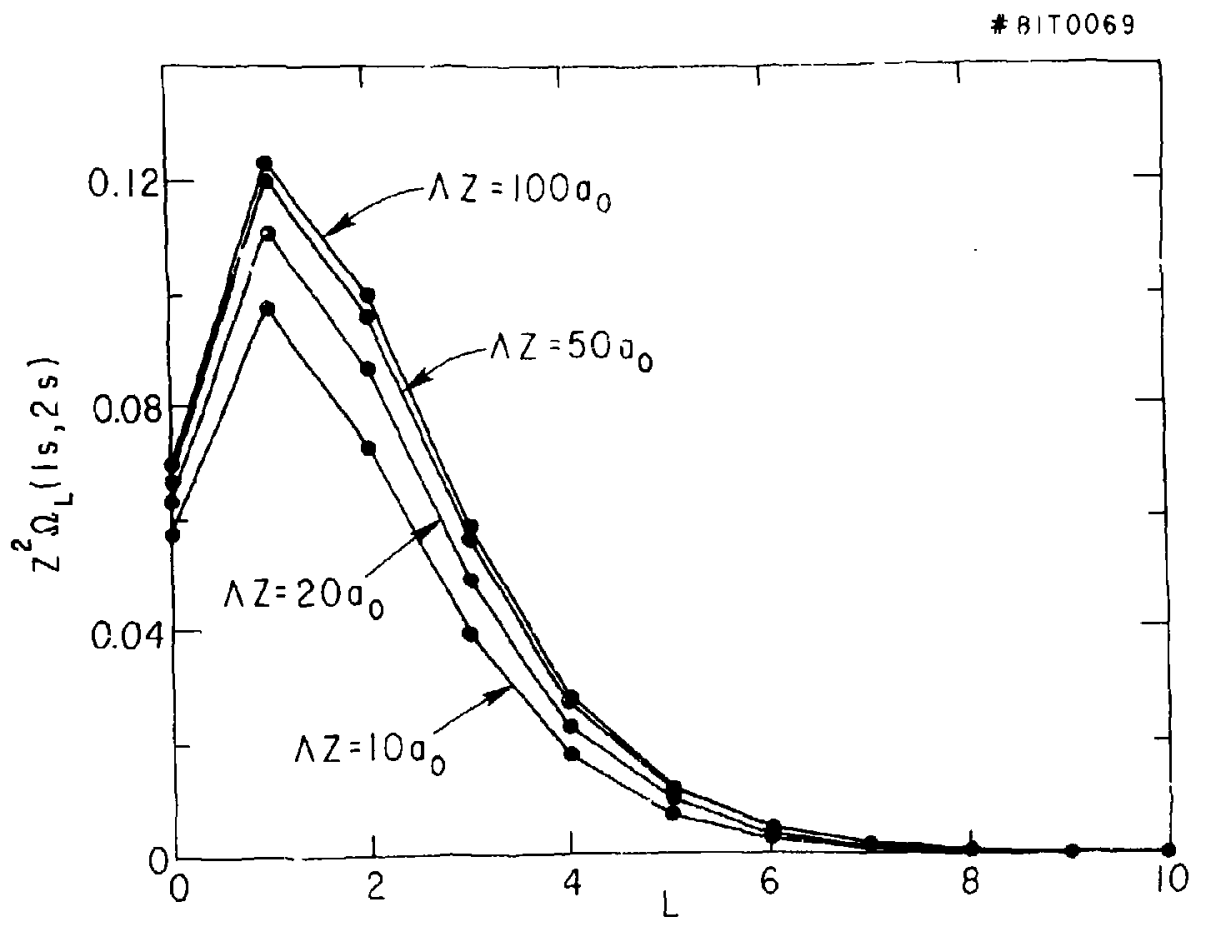




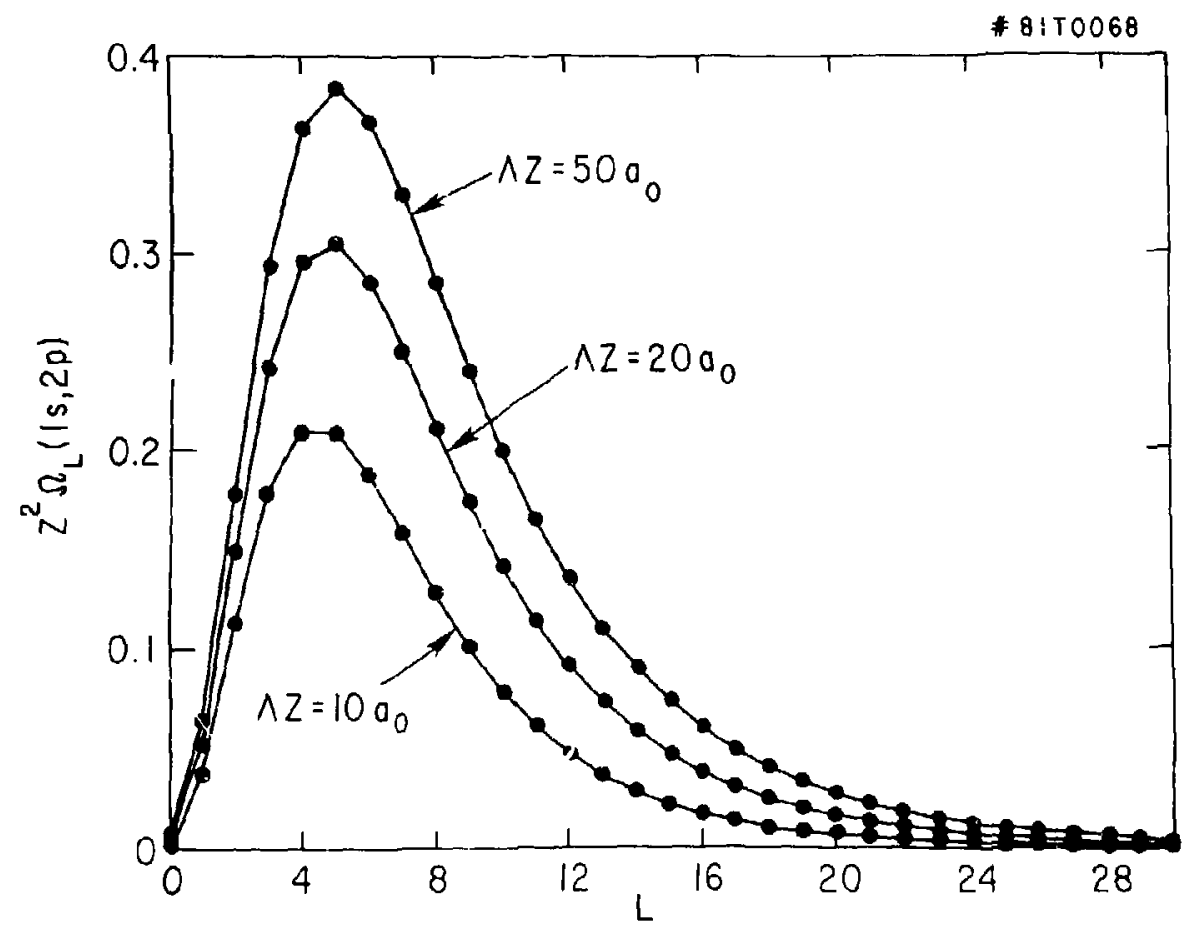




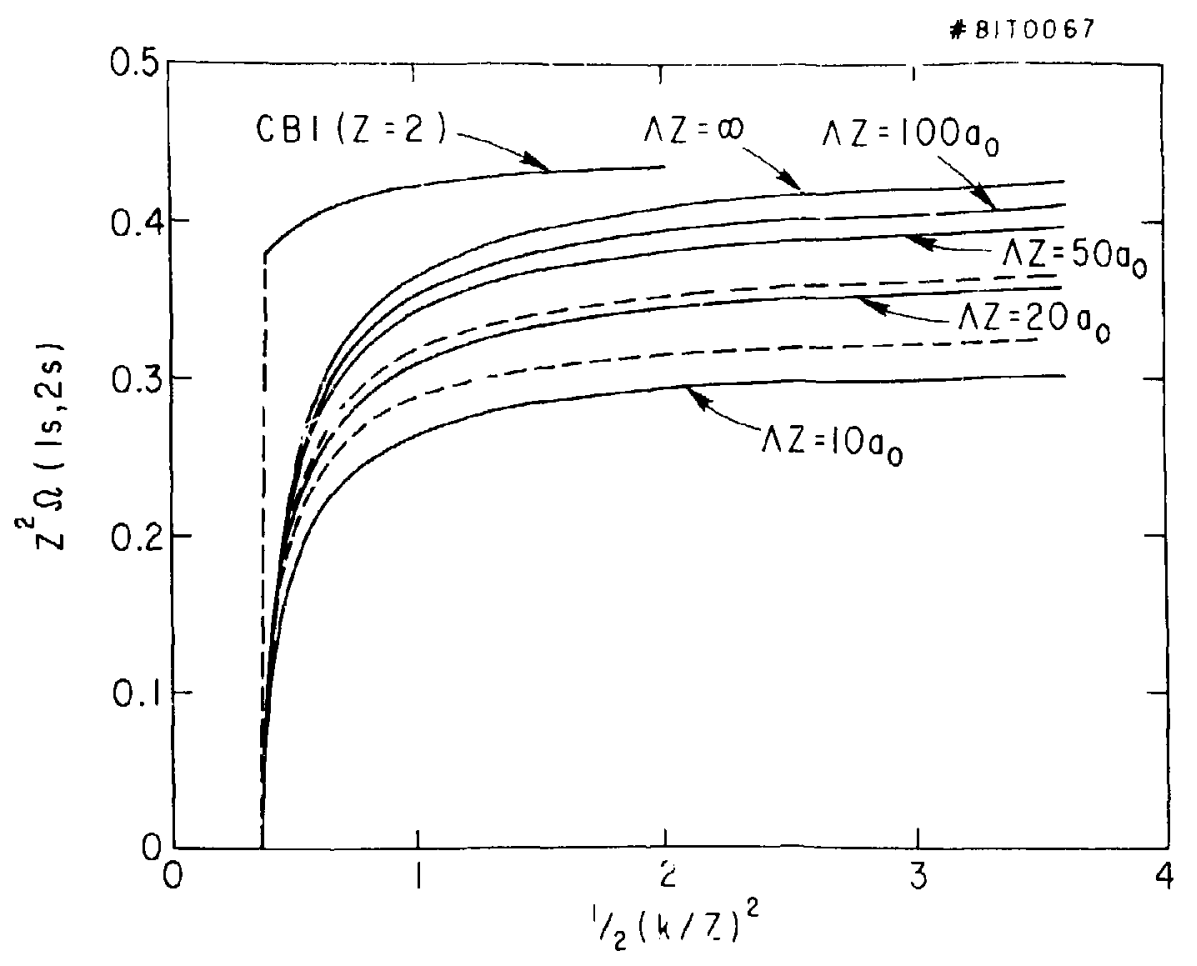




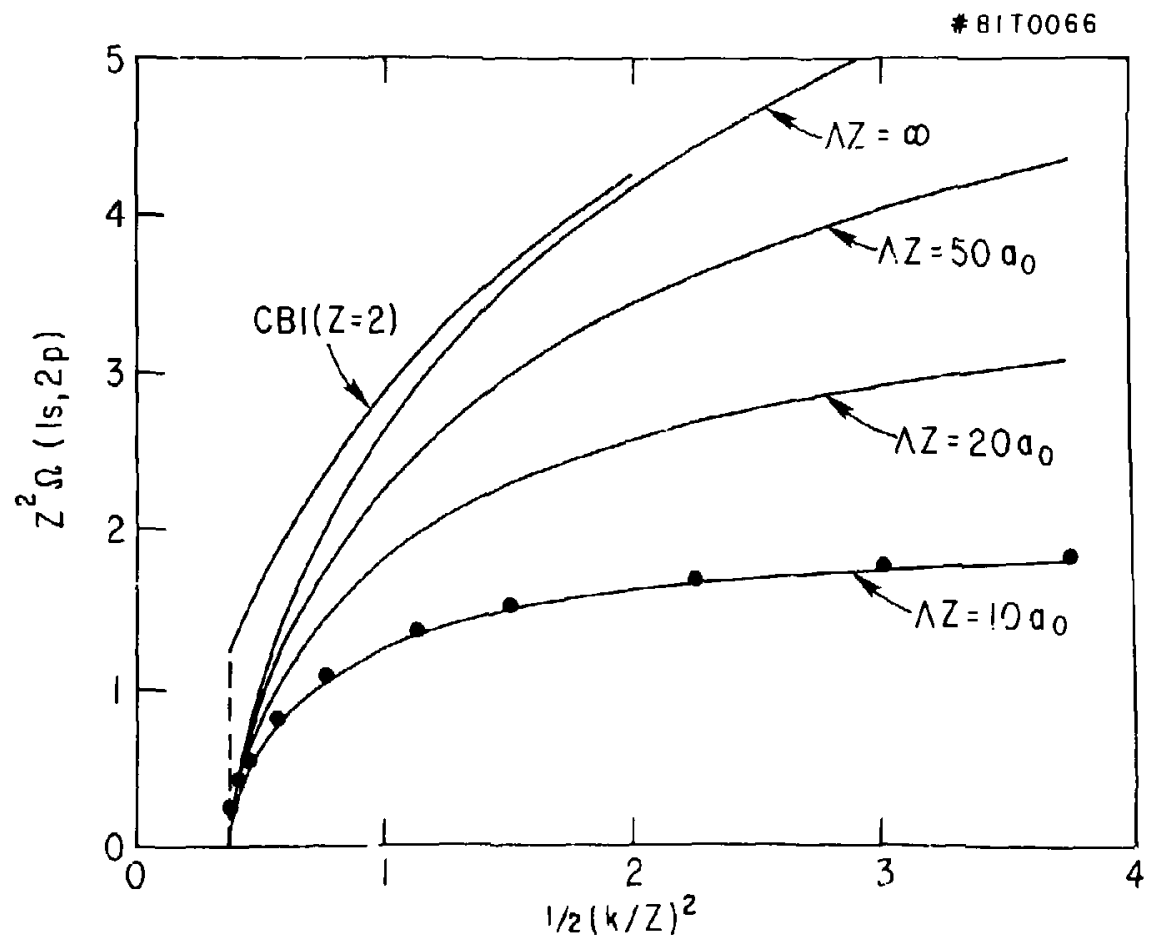




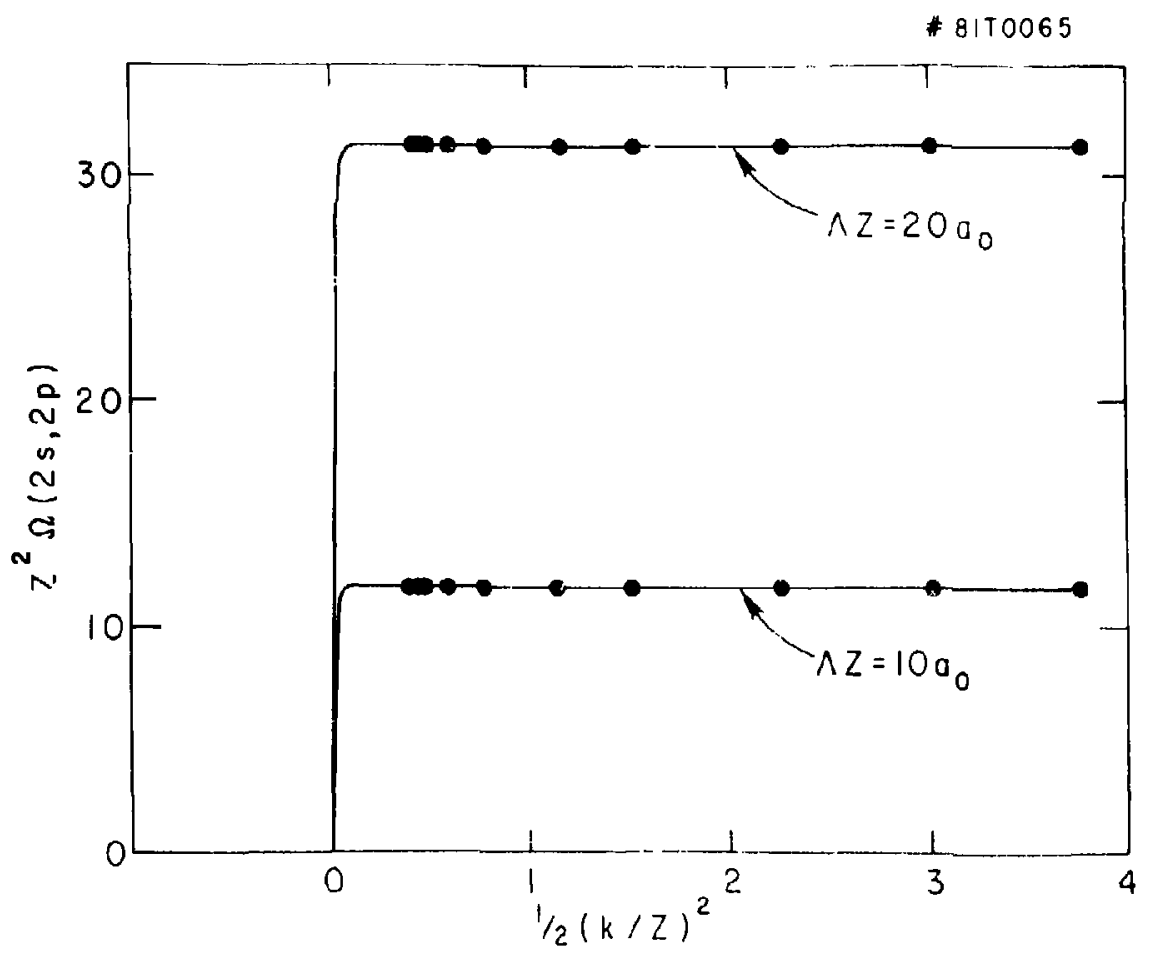

